\title{
Controversies in gestational diabetes
}

\section{Christopher J. Nolan, BMedSci, MBBS, PhD, FRACP, Senior Specialist and Associate Professor*}

Department of Endocrinology and Diabetes, The Canberra Hospital, and the Australian National University Medical School, Canberra, ACT, Australia

\section{Keywords:}

gestational diabetes mellitus

maternal metabolic syndrome

maternal obesity

screening and diagnosis

goals of management

short- and long-term outcomes
Gestational diabetes mellitus (GDM) and controversy are old friends. However, several major studies in the field have clarified some of the main issues. There is now no doubt that hyperglycaemia, at levels less than those that occur in overt diabetes, is associated with adverse pregnancy outcomes, such as large-for-gestational age infants, neonatal hyperinsulinism, neonatal hypoglycaemia and preeclampsia. We also have evidence now that a standard approach to GDM with diagnosis at 24-28 weeks, dietary advice, self-monitoring of blood glucose and insulin therapy as needed reduces these adverse perinatal outcomes. Unknown, however, is if this same approach is effective at reducing long-term risks of metabolic syndrome, type 2 diabetes and cardiovascular disease in both the mothers and babies. For example, could our management strategies miss critical time points of fuel-mediated injury to the foetus important for the baby's long-term metabolic health? The implications of a recent international consensus statement on new diagnostic criteria for GDM are discussed, as well as issues relating to the timing of diagnosis. The potential place for a risk calculator for adverse outcomes in GDM pregnancy that takes into account glycaemic and non-glycaemic risk factors is considered. Such a tool could help stratify GDM women to different levels of care. Ongoing issues relating to maternal glycaemic and foetal growth targets, and the use of oral hypoglycaemic agents in GDM are discussed. To resolve some of the remaining controversies, further carefully designed randomised controlled trials in GDM with long-term follow-up of both mothers and babies are necessary.

(c) 2010 Elsevier Ltd. All rights reserved.

\footnotetext{
* Corresponding author: Department of Endocrinology and Diabetes, The Canberra Hospital, PO Box 11, Woden, ACT 2606, Australia, Tel.: +612 62442228; Fax: +612 62444616.

E-mail address: christopher.nolan@anu.edu.au.
} 
Gestational diabetes mellitus (GDM) is defined as "glucose intolerance with onset or first recognition in pregnancy" or "carbohydrate intolerance of varying severity which is diagnosed in pregnancy and may or may not resolve after pregnancy."1-5 Controversy and GDM have always co-existed. ${ }^{6,7}$ This dates back as far as 1964 when O'Sullivan and Mahan first proposed specific criteria for interpreting the oral glucose tolerance in pregnancy. ${ }^{8}$ The fact that GDM is a very strong risk factor for subsequent permanent diabetes development in the mother has never been a point of contention. ${ }^{9,10}$ The controversy has centred around two questions: (1) Does the maternal hyperglycaemia of GDM pregnancies, independently of associated factors such as obesity and higher maternal age, contribute to adverse pregnancy outcomes? (2) Does the diagnosis and treatment of GDM improve pregnancy outcomes? Recently, there has been substantial progress in resolving these areas of controversy by major studies in the field. The Hyperglycaemia and Adverse Pregnancy Outcomes (HAPO) study, a major international observational study of pregnant women, showed without doubt that maternal hyperglycaemia, less severe than that of diabetes mellitus and corrected for multiple confounders, is associated with several adverse perinatal outcomes. ${ }^{11}$ The Australian Carbohydrate Intolerance Study in Pregnant Women (ACHOIS) and the Maternal-Fetal Medicine Units Network treatment of mild gestational diabetes (MFMUN-GDM) clinical trials showed that diagnosis and treatment of GDM does improve pregnancy outcomes. ${ }^{12,13}$ Controversies continue, however, particularly over the definition of GDM, how best to screen and diagnose the condition, how to deal with the very large number of women affected and how best to manage it cost-effectively, with both the short- and long-term outcomes of the mothers and their babies in mind.

\section{Getting GDM into context}

GDM and the worldwide pandemic of obesity, metabolic syndrome and type 2 diabetes

The increase in the prevalence of type 2 diabetes (T2D) is creating a non-communicable disease global health crisis. ${ }^{14,15}$ The worldwide prevalence of diabetes in 2000 was estimated to be $2.8 \%$, with an expected rise to $4.4 \%$ by 2030 , equating to 366 million persons with diabetes in $2030 .{ }^{14}$ The greatest rises in prevalence are predicted to occur in India, China, Latin America and the Middle East. ${ }^{14}$ This increase in T2D is inextricably linked to the rise in obesity prevalence. ${ }^{16}$ The incidence of GDM is also rising, paralleling the rises in T2D and obesity prevalence. ${ }^{17}$ This is not surprising, considering that GDM and T2D share the same underlying pathogenic mechanisms. They are essentially different phases of the same disease for the mothers. ${ }^{18,19}$ The incidence, however, is difficult to compare from country to country because of differences in screening and diagnosis practices. If the recently published International Association of Diabetes and Pregnancy (IADPSG) recommendations on the diagnosis and classification of hyperglycaemia in pregnancy are adopted, the incidence is expected to be approximately $16-18 \%$ of pregnancies. ${ }^{1}$ Importantly, GDM incidence is likely to have predictive value for future trends in T2D prevalence. Furthermore, GDM also provides an opportunity for timely intervention in mothers and their families to assist in turning this pandemic of metabolic disease around. ${ }^{18}$

\section{Practice points}

- The incidence of GDM is rapidly rising, paralleling closely the pandemic of obesity and T2D.

- GDM incidence could be used as an early barometer for the likely trends in T2D prevalence, particularly if internationally agreed upon criteria of diagnosis are used.

What is the significance of GDM for the woman and her baby during pregnancy?

The HAPO study confirmed in over 23000 women from 15 countries that hyperglycaemia in pregnancy, at levels less than that of diabetes and unknown to the woman or her carers, and adjusted for potential confounders, increases the risk of large-for-gestational age (LGA)/macrosomic babies, 
neonatal hyperinsulinism at birth as reflected by elevated cord C-peptide, neonatal hypoglycaemia, excess neonatal adiposity, shoulder dystocia or birth injury, neonatal hyperbilirubinaemia, primary caesarean section and pre-eclampsia. $11,20,21$ The strong associations between maternal hyperglycaemia, cord blood C-peptide, birth weight and neonatal adiposity are highly consistent with the Pedersen hypothesis. ${ }^{22}$ Pedersen postulated that maternal hyperglycaemia, by increasing glucose passage across the placenta, promotes foetal hyperinsulinism and, this in turn, causes diabetic foetopathy, including increased foetal adiposity and neonatal hypoglycaemia. ${ }^{22}$ HAPO did not show that hyperglycaemia, less than that of overt diabetes, is associated with an increased risk of perinatal mortality. ${ }^{11}$

ACHOIS and the MFMUN-GDM clinical trials have demonstrated that diagnosis and treatment of GDM are worthwhile, as this does reduce the risk of many of the adverse pregnancy outcomes of GDM without causing harm. ${ }^{12,13}$ In both studies, women who had been diagnosed with GDM late in the 2ndearly 3rd trimesters were randomised to either routine care (women and carers were blinded to GDM diagnosis) or intervention. Intervention in both trials consisted of dietary advice, blood glucose monitoring and insulin therapy, as needed. In the ACHOIS study, a composite measure of serious perinatal complications (defined as one or more of death, shoulder dystocia, bone fracture and nerve palsy) was reduced by diagnosis and intervention (adjusted odds ratio (AOR) 0.33, 95\% confidence interval $(\mathrm{CI}) 0.14-0.75, p=0.01) .{ }^{12} \mathrm{~A}$ similar composite measure in the MFMUN-GDM study was reduced, but this was not statistically significant (relative risk $0.87,97 \% \mathrm{CI} 0.72-1.07, p=0.14$ ) ${ }^{13}$ In both studies, rates of LGA/macrosomia and pre-eclampsia were reduced by intervention. ${ }^{12,13}$ Intervention also reduced maternal pregnancy weight gain in both studies. ${ }^{12,13}$ Shoulder dystocia and caesarean section were significantly reduced by treatment in the MFMUN-GDM trial only. ${ }^{13}$ Induction of labour rates and admission to the special care nursery were increased by treatment in the ACHOIS trial only. ${ }^{12}$ Overall, these two trials show that diagnosis and management of GDM with what is a very standard approach can improve pregnancy outcome. . $^{12,13}$

Of particular note, the traditional definition of GDM "glucose intolerance with onset or first recognition in pregnancy" includes women with unknown pre-existing diabetes, particularly T2D. The outcomes of T2D in pregnancy are at least as bad, and may even be worse, than those of type 1 diabetes(T1D) ${ }^{23-25}$ These adverse outcomes include increased rates of congenital malformation and perinatal death. ${ }^{23-25}$ With the rapidly increasing prevalence of T2D in women of childbearing age, undiagnosed T2D in pregnancy is much more common. ${ }^{26,27}$ For this reason, it is time to review the generally accepted definition of GDM and categorise pre-existing overt diabetes recognised for the first time in pregnancy as such rather than GDM. The International Association of Diabetes and Pregnancy Study Groups (IADPSG) Consensus Panel, in its recent statement on the diagnosis and classification of hyperglycaemic disorders in pregnancy recognised this as a major issue. ${ }^{1}$ The panel suggested that a screen for overt diabetes be performed at the first antenatal visit. ${ }^{1}$ However, to reliably prevent congenital malformations in these pregnancies, efforts to diagnose women with overt diabetes prepregnancy need to be pursued.

\section{Practice points}

- Undiagnosed and untreated mild hyperglycaemia in pregnancy is associated with adverse pregnancy outcomes such as LGA infants, neonatal hyperinsulinism, neonatal hypoglycaemia and pre-eclampsia.

- GDM management with a standard approach of dietary advice, blood glucose monitoring and insulin therapy as needed reduces adverse pregnancy outcomes.

- The incidence of undiagnosed pre-existing T2D is increasing and is associated with high rates of serious adverse pregnancy outcomes.

- Early screening for pre-existing T2D needs to become routine practice.

GDM and lifelong health of the mother

Women with GDM are very often on a pathway to the development of T2D. As discussed below, GDM uncovers an inability of the islet $\beta$-cell to compensate for the insulin resistance of pregnancy. ${ }^{28}$ Islet $\beta$-cell failure to compensate for insulin resistance underlies the pathophysiology of T2D. ${ }^{28,29}$ In 
an Australian study of 5470 GDM patients and 783 control subjects, the risk of developing diabetes was 9.6 times greater for patients with GDM. ${ }^{30}$ The cumulative risk of T2D for the GDM patients was $25.8 \%$ at 15 years post diagnosis. ${ }^{30}$ In a systematic review, the cumulative incidence of T2D following GDM pregnancy in 28 studies ranged from $2.6 \%$ to $70 \%$, with factors such as duration of follow-up (6 weeks up to 28 years), diagnostic criteria for GDM used and the rate of retention of subjects in follow-up differing considerably across studies. ${ }^{9}$

GDM is also a risk factor for cardiovascular disease (CVD) events. In a large population based study in Ontario, Canada, women who had GDM in pregnancy $(n=8191)$ compared with a matched control group ( $n=81262$ ) were at higher risk of CVD events with a hazard ratio of $1.71(95 \% \mathrm{CI} 1.08-2.69){ }^{31}$ When adjusted for the subsequent development of T2D, this ratio was no longer significant such that a considerable part of the post-GDM increased CVD risk was attributable to progression to T2D. ${ }^{31}$ Therefore, a diagnosis of GDM for each woman provides an opportunity for intervention to reduce her risk of future T2D and CVD.

\section{Practice points}

- GDM is a strong predictor for the development of T2D and CVD in the mothers.

- This provides an opportunity for diabetes and vascular disease prevention through optimal GDM management during pregnancy and appropriate follow-up management.

\section{GDM and lifelong health of the baby}

There is very strong epidemiological and experimental evidence linking intrauterine growth restriction (IUGR) with later adult diseases such as obesity, hypertension, T2D and CVD. ${ }^{32-34}$ 'Foetal origins of adult disease' is on the agenda of many health-related conferences. Therefore, is there evidence showing that the intrauterine environment of GDM can contribute to adult disease? There is evidence from animal studies, but human data is more limited. ${ }^{35}$

In longitudinal studies of the Pima Indians from Arizona, a population that has an extremely high prevalence of obesity and T2D, established diabetes in mothers during pregnancy does increase the incidence of diabetes in the offspring, particular at young age. ${ }^{36-38}$ An association was found between maternal diabetes in utero and age at offspring's diagnosis of T2D in the multiethnic SEARCH for diabetes in youth study. ${ }^{39}$ Both lines of evidence support the concept that exposure to hyperglycaemia in utero increases the subsequent risk of diabetes in the offspring. The mild hyperglycaemia of GDM, however, was not considered in these studies.

In a predominantly Caucasian population in Denmark, the offspring of women with diet-treated GDM and women with T1D were followed up. ${ }^{40,41}$ At 22 years of age, $21 \%, 11 \%$ and $4 \%$ of the offspring of GDM, T1D and control women, respectively, had T2D or pre-diabetes. ${ }^{41}$ The AORs for T2D/pre-diabetes were 7.8 (95\% CI 2.6-23.4) and 4.0 (95\% CI 1.31-12.3) for the offspring of GDM and T1D women, respectively, compared with the offspring of control women. ${ }^{41}$ The AORs for metabolic syndrome were 4.1 (95\% CI 1.7-10.0) and 2.6 (95\% CI 1.0-6.5) for the offspring of diet-treated GDM and T1D. ${ }^{40}$ Clearly, offspring of women with GDM are at a significantly higher risk of overweight and T2D. A prevalence of $21 \%$ of $\mathrm{T} 2 \mathrm{D} /$ pre-diabetes at the age of 22 years is very high and concerning. While it is difficult to determine the relative contributions of the intrauterine environment compared with genes and the post-pregnancy environment on these findings in the children of GDM women, the same trends in the children of T1D women strongly indicate the hyperglycaemia of the intrauterine environment as being important. This conclusion is reinforced by the finding of an association between maternal glucose control late in pregnancy and the risk of T2D/pre-diabetes in the offspring of the T1D mothers. ${ }^{41}$

Of concern in the Danish study, the children of the women with diet-treated GDM did not do well in follow-up with respect to the development of overweight and hyperglycaemia. ${ }^{40,41}$ The questions remain: Can optimal management of GDM during pregnancy reduce the risk of metabolic syndrome and diabetes in the offspring later in life? If so, what is the optimal management protocol? Can we do more after pregnancy (e.g., during the neonatal period and early childhood) to reduce the long-term risk for the offspring? 


\section{Practice points}

- GDM is associated with a substantially increased risk of metabolic syndrome and T2D in the offspring later in life.

- The optimal approach during pregnancy and in postnatal life to reduce this long-term risk is unknown.

\section{Goals to our approach to GDM}

Considering the above, our overall goals in approaching GDM should be the following:

- To prevent adverse pregnancy outcome for the baby and the mother;

- To promote lifelong health for the baby and the mother; and

- To enhance the overall metabolic health of current and future generations.

\section{Pathophysiology of GDM}

GDM is a condition of failure of the islet $\beta$-cell to compensate for insulin resistance

For the majority, the occurrence of GDM is an indication of an underlying risk for T2D. A small percentage of women with GDM, however, will have early T1D or maturity onset diabetes of the young (MODY). ${ }^{42,43}$ It is important to be aware of these possibilities, if women with GDM have atypical presentations. For example, early T1D needs to be considered in lean women with relatively high blood sugar levels. MODY needs to be considered in non-obese pregnant women with strong family histories of early-onset non-insulin-requiring diabetes. The following discussion of pathophysiology, however, concerns the majority of GDM women at risk of T2D.

The hyperglycaemia in women with GDM develops due to a failure of islet $\beta$-cells to sustain compensatory insulin secretion for insulin resistance. ${ }^{29,44}$ Many of the women destined to develop diabetes will have had insulin-resistance prepregnancy usually related to overweight and obesity. ${ }^{45,46}$ Maternal adaptation to pregnancy also includes a physiological induction of insulin resistance that will aggravate any pre-existing insulin resistance. ${ }^{45,46}$ Thus, pregnancy-induced aggravation of insulin resistance exerts a major load on islet $\beta$-cells, and women with an underlying susceptibility to islet $\beta$ cell failure will develop GDM. ${ }^{44}$

Considering that islet $\beta$-cell failure to insulin resistance is a necessary factor in the pathogenesis of T2D, it is no surprise that GDM predicts it so well. ${ }^{29}$ Pregnancy-induced insulin resistance can, therefore, be considered to be an islet $\beta$-cell stress test for the prediction of T2D risk.

What is the relative contribution of obesity and hyperglycaemia to adverse pregnancy outcomes in GDM pregnancy?

The HAPO study showed that even mild degrees of hyperglycaemia, after adjustment for confounding factors including maternal body mass index (BMI), are associated with greater risk of LGA babies, clinical neonatal hypoglycaemia and raised cord blood C-peptide. ${ }^{11}$ From the same study, higher maternal BMI, independent of maternal hyperglycaemia, was also strongly associated with excess foetal growth and adiposity, and increased pre-eclampsia. ${ }^{47}$ Thus, hyperglycaemia and high BMI are independent risk factors for adverse pregnancy outcome.

In a recent large Australian study, the AORs of being obese or morbidly obese for hypertensive disorders of pregnancy were 3.0 and 4.9; for GDM, 3.0 and 7.4; for hospital stay greater than 5 days, 1.5 and 3.2; and for caesarean section, 2.0 and $2.3 .{ }^{48}$ For the neonates, AOR of maternal obesity or morbid obesity for birth defects were 1.6 and 3.4, and for hypoglycaemia 2.6 and 7.1. ${ }^{48}$

With respect to birth defects, a prospective cohort study (22 951 pregnancies) of early pregnancy exposures and pregnancy outcome in the UK showed that women who were both obese and diabetic were 3.1 times more likely to have a baby with a major congenital malformation compared with 
non-obese, non-diabetic women. ${ }^{49}$ Major congenital malformations, however, were not increased in pregnancies complicated by obesity alone or diabetes alone. ${ }^{49}$ It is highly likely, therefore, that obesity and diabetes synergise in causing adverse pregnancy outcomes.

Is excessive maternal weight gain a major confounding risk factor for adverse outcome in GDM pregnancy?

Excess weight gain in obese women, irrespective of glycaemic status, has been linked to adverse pregnancy outcome. ${ }^{50-52}$ Furthermore, higher maternal weight gain increases the risk of macrosomia in women with hyperglycaemia, such that the highest macrosomia rates are in women with the combination of gestational hyperglycaemia and excessive pregnancy weight gain. ${ }^{53}$ Thus, excessive weight gain is an additional risk factor for adverse outcomes in GDM pregnancy.

What is the relative importance of hyperglycaemia compared with the non-glycaemic components of the metabolic syndrome on pregnancy outcome?

The metabolic syndrome is a complex of interrelated risk factors for conditions such as non-alcoholic fatty liver disease (NAFLD), polycystic ovarian syndrome (PCOS) and cardiovascular disease as well as GDM and T2D. ${ }^{54-58}$ These risk factors include dysglycaemia, elevated triglyceride, low high-density lipoprotein (HDL)-cholesterol, raised blood pressure and central obesity. ${ }^{56,57}$ Other non-glycaemic factors associated with the metabolic syndrome include hyperinsulinaemia, elevated non-esterified fatty acids (NEFAs), hypoadipinectinaemia, a prothrombotic state and a pro-inflammatory state. ${ }^{57,59,60}$ Therefore, how important are the non-glycaemic factors and their interaction with elevated glucose?

Metabolic syndrome in early pregnancy has been shown to increase adverse pregnancy outcomes. For example, it increases the risk of pre-term birth. ${ }^{61}$ Altered adipocytokine levels measured early in pregnancy, known to occur in subjects with metabolic syndrome, are also associated with higher rates of pre-eclampsia. ${ }^{62}$ It also works the other way; pre-eclampsia and GDM are risk factors for postpartum metabolic syndrome. ${ }^{63,64}$

What about lipids? Normal maternal metabolism during pregnancy is characterised by a physiological hyperlipidaemia. ${ }^{65,66} \mathrm{GDM}, \mathrm{T} 2 \mathrm{D}$ and poorly controlled T1D, however, are often associated with even higher blood lipids than in normal pregnancy. ${ }^{66-68}$ Obese women have abnormal lipid profiles in pregnancy with a pattern of hypertriglyceridaemia, elevated very low density lipoprotein (VLDL)cholesterol and low HDL-cholesterol, in association with hyperinsulinaemia, increased inflammatory markers and increased leptin. ${ }^{69}$ Women with higher range NEFA in the 3rd trimester are more likely to be overweight or obese. ${ }^{70}$ Elevated NEFA have been shown to be associated with pre-term delivery, IUGR, IUGR with pre-eclampsia and foetal adiposity. ${ }^{70-72}$ Whether elevated NEFA levels have a pathogenic role in these adverse events, or simply correlate with other metabolic syndrome pathogenic factors, however, is unknown.

Thus, the women we treat with GDM often have additional risk factors (confounders) for adverse pregnancy outcome. These include maternal obesity, dyslipidaemia, other metabolic syndrome-related factors and increased maternal age. These and other additional risk factors may act independently and/ or synergistically to cause harm.

Does the GDM impact on short- and long-term health of the baby occur predominantly in the late 2nd and 3rd trimesters or does the impact start earlier?

The current paradigm used for the diagnosis and management of GDM is founded partly on O'Sullivan's initial use of oral glucose tolerance tests (OGTTs) in middle to late pregnancy to predict diabetes after pregnancy and on Freinkel's fuel-mediated teratogenesis hypothesis in which he predicted that GDM causes harm to the baby only in the second half of pregnancy. ${ }^{73,74}$ However, relative mild hyperglycaemia and hyperlipidaemia are almost certainly present in many GDM women from an earlier stage of pregnancy, and obesity is definitely present in many from earlier on. The ACHOIS study and the MFMUN-GDM study do show that the use of this paradigm is effective in reducing adverse perinatal outcomes. ${ }^{12,13}$ However, is diagnosing GDM at 24-28 weeks and managing it in the later part 
of pregnancy adequate to prevent the long-term harmful effects on the offspring? We do not have the answer to this important question.

\section{Practice points}

- The hyperglycaemia of GDM develops due to failure of the islet $\beta$-cell to sustain compensatory insulin secretion for the aggravated insulin resistance of pregnancy.

- Pregnancy can be considered to be an islet $\beta$-cell stress test.

- Obesity has been shown to be a risk factor, independent of glucose, for adverse pregnancy outcomes.

- Hyperlipidaemia and other non-glycaemic factors associated with the metabolic syndrome may also contribute to adverse pregnancy outcomes in GDM pregnancy.

- The critical time points of long-term fuel-mediated injury to the foetus in GDM pregnancy are unknown.

\section{Screening and diagnosis of GDM: the IADPSG consensus statement on the diagnosis of hyperglycaemia in pregnancy}

Until recently, there has been a lack of international consensus on the screening and diagnosis of GDM. The various diagnostic criteria used were not based on the prediction of adverse pregnancy outcomes, but on the prediction of the risk of diabetes after pregnancy or on the OGTT diagnostic criteria for impaired glucose tolerance and diabetes in the non-pregnant population. ${ }^{1-5,75}$ The HAPO study was designed to determine the actual levels of blood glucose during a 75-g OGTT performed between 24 and 32 weeks of pregnancy, lower than that of overt diabetes, that predicted adverse perinatal outcomes. ${ }^{1,11}$ The study showed a continuous relationship between maternal glycaemia and the adverse pregnancy outcomes of LGA babies, primary caesarean section, clinically defined hypoglycaemia and cord C-peptide $>90$ th percentile. ${ }^{11}$ There was no inflection point for any of the relationships. ${ }^{11}$ It is important to also add that the HAPO findings are consistent with other studies that show a relationship between mildly elevated blood glucose levels and adverse outcome. ${ }^{76-79}$ To translate the HAPO study data into clinical practice, an IADPSG consensus panel (with representatives from the 10 member organisations) met in 2008 to make recommendations on the diagnosis and classification of hyperglycaemia in pregnancy. ${ }^{1}$ Due to the continuum of risk, the IADPSG panel based its decision on an agreed but arbitrary decision about ORs. Thresholds chosen for the diagnostic cut-off values for the fasting, $1 \mathrm{~h}$ and $2 \mathrm{~h}$ glucose levels were based on a fully adjusted OR of 1.75 for birth weight $>90$ th percentile, cord C-peptide $>90$ th percentile and percent body fat $>90$ th percentile. ${ }^{1}$ The IADPSG diagnostic criteria for GDM are shown in Table 1.

Important points to make about the IADPSG consensus statement on the diagnosis of GDM are the following. Selective screening based on risk factors for GDM has not been recommended. Screening by a glucose challenge test (GCT) is not recommended, as the GCT does not detect women with fasting hyperglycaemia only. Thus, all women without an earlier diagnosis of GDM or diabetes are recommended to have a $2 \mathrm{~h}$ OGTT at 24-28 weeks of pregnancy. Based on the HAPO cohort, the proposed IADPSG diagnostic criteria will diagnose about $17.8 \%$ of women with a hyperglycaemic disorder of pregnancy. ${ }^{1}$

As discussed above, the IADPSG panel also recommended a screening test to be performed at the first prenatal visit to avoid late diagnosis of pre-existing overt diabetes (Table 1). ${ }^{1}$ The panel stated that this could be for all women or could be a selective screen in high-risk women. ${ }^{1}$ Many of the IADPSG panel favoured using the HbA1c test for this screen, but the use of fasting plasma glucose or random blood glucose was suggested as alternative options if HbA1c testing is not feasible. ${ }^{1} \mathrm{~A}$ HbA1c $\geq 6.5 \%$ is now accepted to be diagnostic of diabetes in non-pregnant individuals by the American Diabetes Association, with the range of $5.7-6.4 \%$ being considered to be indicative of pre-diabetes. ${ }^{80}$ The IADPSG panel suggested a screening fasting plasma glucose $\geq 5.1$, but a value $<7.0 \mathrm{mmol}^{-1}$ is diagnostic of GDM; however, they did not suggest HbA1c levels for diagnosis of GDM on the first antenatal visit screen. ${ }^{1}$

The recommended IADPSG approach to diagnosis and classification of hyperglycaemic disorders of pregnancy is a major advance. First, the new diagnostic criteria are linked to the risk of adverse pregnancy 
Table 1

IADPSG diagnostic criteria for GDM and overt diabetes in pregnancy. ${ }^{1}$

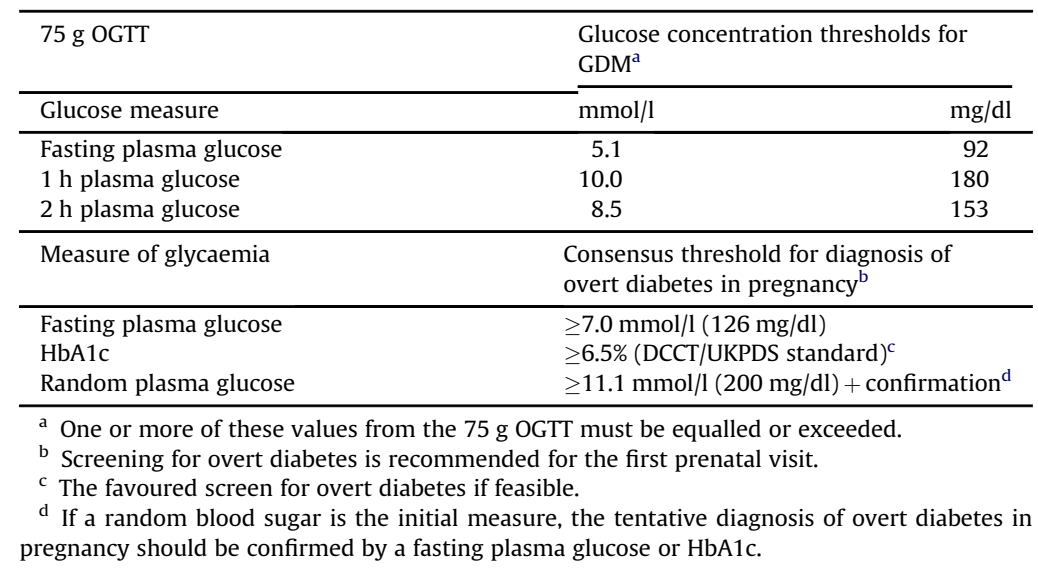

outcomes. Second, the issue of late diagnosis of overt diabetes in pregnancy has been addressed. Third, it promotes a uniform approach to enable international comparison of GDM prevalence and pregnancy outcomes. A very significant issue that needs to be addressed by all health services adopting these recommendations, however, is the increased numbers of women that will be diagnosed (up to a doubling depending on old criteria used and local prevalence). This will place enormous strain on health-service personnel and costs, if not carefully addressed. It is important to ask, "Can the women diagnosed with GDM be stratified into low-risk and high-risk groups?" Low-risk women could receive lifestyle advice and usual pregnancy care only, whereas high-risk women could follow current management approaches. As discussed above, factors other than glycaemia can also contribute to the risk of adverse outcome. The development of a risk calculator that takes into consideration multiple risk factors on adverse pregnancy outcome such as the number of abnormal values on the OGTT, obesity, higher maternal age and ethnicity could prove very useful in stratifying GDM risk to determine the level of pregnancy care required.

\section{Practice points}

- The IADPSG consensus panel recommendations for GDM diagnosis are based on the risk of hyperglycaemia on adverse pregnancy outcome.

- Screening at the first antenatal visit to diagnose overt diabetes early in pregnancy is recommended.

- The IADPSG criteria may diagnose up to $18 \%$ of women with GDM, depending on local demographics.

- The IADPSG recommendations do not take into consideration factors other than glucose such as obesity that increase the risk of adverse pregnancy outcome.

- Risk stratification of women with GDM is likely to be necessary for health services adopting the IADPSG recommendations.

- Risk calculator development that includes glycaemic and non-glycaemic risk factors for adverse pregnancy outcomes in GDM women should be considered.

\section{Management of GDM: is our current approach improving both the short- and long-term outcomes?}

There has been major progress in recent years with respect to the management of GDM. The ACHOIS and MFMUN-GDM studies show that the standard approach of diagnosis at 24-32 weeks, dietary advice, self-monitoring of blood glucose and insulin therapy, as needed, can improve short-term 
(perinatal) outcomes. ${ }^{12,13}$ Randomised controlled trials (RCTs) of the use of glibenclamide (glyburide) (published 2000) and metformin (published 2008) versus insulin in GDM pregnancy were favourable for their use with respect to perinatal outcomes. ${ }^{81,82}$ None of the above studies, however, considered long-term outcomes. Other chapters within this series focus on oral agents, insulin, insulin analogues and obstetric management, such that the following discussion will mainly relate to optimising management for both short- and long-term outcomes for mothers and babies. Unfortunately, more questions are raised than answers given.

\section{Timing of GDM diagnosis and management}

As discussed above, we currently diagnose most women with GDM in the late-second and earlythird trimesters. This does provide a window of opportunity to reduce the rates of macrosomia and pre-eclampsia as was shown in the ACHOIS and MFMUN-GDM studies. ${ }^{12,13}$ However, we do not know if this is early enough to reduce longer-term risk of obesity, metabolic syndrome and T2D in the offspring. What is the critical developmental stage that determines the long-term metabolic health of adipose tissue, muscle insulin sensitivity and the pancreatic islet $\beta$-cell? If it is earlier than 28 weeks, we may be too late with our current approach to improve the long-term health of the baby. Do we need to start focussing our diagnosis and management strategy earlier in pregnancy?

\section{Management targets in GDM}

The 5th International Workshop Conference on GDM made recommendations relating to targets for glycaemia during GDM pregnancy, and the potential role of foetal growth targets. ${ }^{75}$ The workshop recommended maintaining capillary blood glucose at $<5.3 \mathrm{mmoll}^{-1}\left(<96 \mathrm{mg} \mathrm{dl}^{-1}\right)$ in the fasting state, $<7.8 \mathrm{mmol}^{-1}\left(<140 \mathrm{mg} \mathrm{dl}^{-1}\right)$ at $1 \mathrm{~h}$ and $<6.7 \mathrm{mmoll}^{-1}\left(<120 \mathrm{mg} \mathrm{dl}^{-1}\right)$ at $2 \mathrm{~h}$ after starting a meal. ${ }^{75}$ These targets were based on the then knowledge of normal glycaemia in pregnancy and the outcomes of the ACHOIS study. ${ }^{75}$ They commented that data from controlled trials of lower versus higher targets were lacking. ${ }^{75}$ Careful analysis of the metformin versus insulin in gestational diabetes study (MiG study) showed a strong association between the level of glycaemia achieved and pregnancy outcomes, such that the authors commented that lower glycaemic targets may be necessary. ${ }^{79}$ The 5 th International Workshop stated that evidence from RCTs indicated that modification of metabolic management based on foetal growth measurements by ultrasound (particularly abdominal circumference $<75$ th percentile) may improve perinatal outcome. ${ }^{75}$ Therefore, we do have some idea about targets for short-term outcomes, but what about for long-term outcomes? For a GDM baby tracking on the 95th percentile for body weight at 28 weeks, should our management strategies be targeting birth weight $<75$ th percentile, necessitating a substantial correction, or should we be aiming for $<90$ th percentile? We know that IUGR can increase the risk of subsequent metabolic syndrome and diabetes; therefore, can too aggressive correction of foetal growth over the last few weeks of GDM pregnancy do the same? To answer this, we do need RCTs of tight versus less tight management strategies with long-term follow-up of the babies. In the meantime, the recommendations of the 5th International Workshop are the best we have and seem appropriate.

Should we use oral agents?

If proven to be both effective and safe, the use of oral hypoglycaemic agents in GDM will be a major advance. While this topic is covered in another chapter of this series, it is worth making some cautionary comment here with respect to the use of these agents and long-term outcomes of GDM pregnancy. Unfortunately, we do not have RCTs of oral agents versus insulin on long-term outcomes of mothers or babies. The two agents that are favoured for use in GDM pregnancy, based on perinatal outcome studies, are glibenclamide (glyburide) and metformin.

Glibenclamide is a sulphonylurea that acts as an insulin secretagogue. There is some controversy over whether it can cross the placenta. ${ }^{83,84}$ From studies in non-pregnant individuals, questions have been raised about the safety of glibenclamide for the islet $\beta$-cell, such that it could potentially harm the maternal and foetal $\beta$-cells if used in pregnancy. ${ }^{29,85}$ Therefore, we do need to know if this drug has any long-term harmful effects on the mother and baby before being able to fully endorse its use in GDM pregnancy. 
Metformin is now accepted to be the first-line oral hypoglycaemic agent for T2D in non-pregnant patients. Its mechanism of action is not fully understood, but it may involve activation of adenosine monophosphate (AMP)-activated protein kinase, a key enzyme involved in the regulation of cellular energy metabolism. ${ }^{86,87}$ Metformin easily crosses the placenta such that it has the potential to affect foetal development. While the MiG study suggested that metformin does not cause harm to the baby in the perinatal period, we really do not know yet if it has detrimental or beneficial long-term effects. Of potential benefit to the mother over insulin, metformin use was associated with lower weight gain in the MiG study ${ }^{81}$ This could translate to less-severe overweight/obesity and T2D for the mothers later on. RCTs with long-term maternal and baby follow-up of GDM mothers are again required for metformin.

\section{Lifestyle in management of GDM}

Lifestyle change is perhaps the most important component of management in GDM pregnancy. Very often, women make substantial change to their diet after diagnosis (at 26-32 weeks), and this is very noticeable to them on self-blood glucose monitoring. Exercise change is also important, but more difficult to achieve in the short term. While it is clear that RCTs are required to determine the optimal healthy diet (e.g., optimal carbohydrate type and content), diets comprising large quantities of junk food are indisputably bad. ${ }^{88,89}$ To be delaying efforts at improvement of lifestyle until after GDM diagnosis of pregnancy at 24-32 weeks of pregnancy is almost certainly lost opportunity. Efforts to improve the lifestyle of women at risk of GDM, both prepregnancy and from early pregnancy need to be considered. Such an approach has the potential of both preventing GDM as well as improving overall short- and long-term outcomes for the mothers and babies.

\section{Practice points}

- The standard strategies for the management of GDM used in the ACHOIS and MFMUN-GDM studies are effective in reducing adverse perinatal outcomes.

- It is unknown if these strategies will also be effective in improving long-term outcomes for the mothers and babies of GDM pregnancies.

\section{Follow-up of women with GDM}

It is known that lifestyle and pharmacological measures can reduce the conversion of impaired glucose tolerance to diabetes (with some subjects being post GDM), but this is within major clinical trials with substantial resources available to maintain compliance. ${ }^{90-94}$ We really do not know how to develop successful programmes of follow-up and prevention of future T2D in post-GDM women within the usual practice setting in which resources are very limited. Efforts to prevent T2D in the mothers need to be family focussed, including a strategy to prevent obesity and diabetes in the children as well.

\section{Research agenda}

- To develop a risk calculator taking into account glycaemic and non-glycaemic risk factors to help stratify women with GDM to various levels of care.

- To determine if the timing of diagnosis and management of GDM is early enough to reduce the adverse effects of the GDM intrauterine environment on the long-term health of the baby.

- To determine the maternal glycaemic and foetal growth targets for optimal short- and longterm outcomes of GDM pregnancy.

- To determine if oral agents are safe for the long-term health of mothers and babies.

- To determine strategies for the prevention of GDM and the prevention of subsequent obesity, T2D and CVD in the mothers.

- To determine strategies post birth to improve the long-term health of the babies. 


\section{Conflict of interest statement}

Associate Professor Christopher Nolan has received speaker fees and funding for travel to conferences related to this field from Novo Nordisk.

\section{References}

1. International Association of Diabetes in Pregnancy Groups Consensus Panel. International association of diabetes and pregnancy study groups recommendations on the diagnosis and classification of hyperglycemia in pregnancy. Diabetes Care 2010; 33: 676-682.

2. National Collaborating Centre for Women's and Children's Health. Diabetes in pregnancy: management of diabetes and its complications from preconception to the postnatal period (Commissioned by National Institute of Health and Clinical Excellence), http://wwwniceorguk/nicemedia/pdf/CG063FullGuidelinepdf; 2008.

3. American Diabetes Association. Diagnosis and classification of diabetes mellitus. Diabetes Care 2006; 29(Suppl. 1): S43-S48.

4. IDF Clinical Guidelines Task Force. Global guideline on pregnancy and diabetes, http://wwwidforg/webdata/docs/ Pregnancy_EN_RTPpdf; 2009.

5. Hoffman L, Nolan C, Wilson JD et al. Gestational diabetes mellitus-management guidelines. The Australasian Diabetes in Pregnancy Society. Med J Aust 1998; 169: 93-97.

6. Jarrett RJ. Gestational diabetes: a non-entity? BMJ 1993; 306: 37-38.

7. Jarrett RJ. Reflections on gestational diabetes mellitus. Lancet 1981; 2: 1220-1221.

8. O'Sullivan JB \& Mahan CM. Criteria for the oral glucose tolerance test in pregnancy. Diabetes 1964; 13: $278-285$.

9. Kim C, Newton KM \& Knopp RH. Gestational diabetes and the incidence of type 2 diabetes: a systematic review. Diabetes Care 2002; 25: 1862-1868.

10. Bellamy L, Casas JP, Hingorani AD et al. Type 2 diabetes mellitus after gestational diabetes: a systematic review and metaanalysis. Lancet 2009; 373: 1773-1779.

11. Metzger BE, Lowe LP, Dyer AR et al. Hyperglycemia and adverse pregnancy outcomes. N Engl J Med 2008; 358: 1991-2002.

12. Crowther CA, Hiller JE, Moss JR et al. Effect of treatment of gestational diabetes mellitus on pregnancy outcomes. $N$ Engl $J$ Med 2005; 352: 2477-2486.

13. Landon MB, Spong CY, Thom E et al. A multicenter, randomized trial of treatment for mild gestational diabetes. $N$ Engl J Med 2009; 361: 1339-1348.

14. Wild S, Roglic G, Green A et al. Global prevalence of diabetes: estimates for the year 2000 and projections for 2030. Diabetes Care 2004; 27: 1047-1053.

15. Zimmet P, Alberti KG \& Shaw J. Global and societal implications of the diabetes epidemic. Nature 2001; 414: $782-787$.

16. Colagiuri S. Diabesity: therapeutic options. Diabetes Obes Metab 2010; 12: 463-473.

17. Anna V, van der Ploeg HP, Cheung NW et al. Sociodemographic correlates of the increasing trend in prevalence of gestational diabetes mellitus in a large population of women between 1995 and 2005. Diabetes Care 2008; 31: 2288-2293.

18. England LJ, Dietz PM, Njoroge T et al. Preventing type 2 diabetes: public health implications for women with a history of gestational diabetes mellitus. Am J Obstet Gynecol 2009; 200(365): e361-e368.

19. Kim SY, England L, Wilson HG et al. Percentage of gestational diabetes mellitus attributable to overweight and obesity. Am J Public Health 2010; 100: 1047-1052.

20. The HAPO Study Cooperative Research Group. Hyperglycemia and Adverse Pregnancy Outcome (HAPO) Study: associations with neonatal anthropometrics. Diabetes 2009; 58: 453-459.

21. Yogev, Chen, Hod et al. Hyperglycemia and Adverse Pregnancy Outcome (HAPO) study: preeclampsia. Am J Obstet Gynecol 2010; 202(255): e251-e257.

22. Pedersen J. Diabetes and pregnancy. Blood sugar of newborn infants. Copenhagen: Danish Science Press, 1952.

23. McElduff A, Ross GP, Lagstrom JA et al. Pregestational diabetes and pregnancy: an Australian experience. Diabetes Care 2005; 28: 1260-1261.

24. Cundy T, Gamble G, Townend K et al. Perinatal mortality in Type 2 diabetes mellitus. Diabet Med 2000; 17: 33-39.

25. Clausen TD, Mathiesen E, Ekbom Pet al. Poor pregnancy outcome in women with type 2 diabetes. Diabetes Care 2005; 28: 323-328.

26. Lawrence JM, Contreras $\mathrm{R}$, Chen $\mathrm{W}$ et al. Trends in the prevalence of preexisting diabetes and gestational diabetes mellitus among a racially/ethnically diverse population of pregnant women, 1999-2005. Diabetes Care 2008; 31: 899-904.

27. Dunstan DW, Zimmet PZ, Welborn TA et al. The rising prevalence of diabetes and impaired glucose tolerance: the Australian Diabetes, Obesity and Lifestyle Study. Diabetes Care 2002; 25: 829-834.

28. Buchanan TA, Xiang A, Kjos SL et al. What is gestational diabetes? Diabetes Care 2007; 30(Suppl. 2): S105-S111.

29. Prentki M \& Nolan CJ. Islet beta cell failure in type 2 diabetes. J Clin Invest 2006; 116: 1802-1812.

30. Lee AJ, Hiscock RJ, Wein P et al. Gestational diabetes mellitus: clinical predictors and long-term risk of developing type 2 diabetes: a retrospective cohort study using survival analysis. Diabetes Care 2007; 30: 878-883.

31. Shah BR, Retnakaran R \& Booth GL. Increased risk of cardiovascular disease in young women following gestational diabetes mellitus. Diabetes Care 2008; 31: 1668-1669.

32. Barker DJ, Hales CN, Fall CH et al. Type 2 (non-insulin-dependent) diabetes mellitus, hypertension and hyperlipidaemia (syndrome X): relation to reduced fetal growth. Diabetologia 1993; 36: 62-67.

33. Mi J, Law C, Zhang KL et al. Effects of infant birthweight and maternal body mass index in pregnancy on components of the insulin resistance syndrome in China. Ann Intern Med 2000; 132: 253-260.

34. Simmons R. Developmental origins of adult metabolic disease: concepts and controversies. Trends Endocrinol Metab 2005; 16: $390-394$.

35. Aerts L \& Van Assche FA. Intra-uterine transmission of disease. Placenta 2003; 24: 905-911.

36. Dabelea D \& Pettitt DJ. Intrauterine diabetic environment confers risks for type 2 diabetes mellitus and obesity in the offspring, in addition to genetic susceptibility. J Pediatr Endocrinol Metab 2001; 14: 1085-1091. 
37. Pettitt DJ, Aleck KA, Baird HR et al. Congenital susceptibility to NIDDM. Role of intrauterine environment. Diabetes 1988; 37: 622-628.

38. Franks PW, Looker HC, Kobes S et al. Gestational glucose tolerance and risk of type 2 diabetes in young Pima Indian offspring. Diabetes 2006; 55: 460-465.

39. Pettitt DJ, Lawrence JM, Beyer J et al. Association between maternal diabetes in utero and age at offspring's diagnosis of type 2 diabetes. Diabetes Care 2008; 31: 2126-2130.

40. Clausen TD, Mathiesen ER, Hansen T et al. Overweight and the metabolic syndrome in adult offspring of women with diettreated gestational diabetes mellitus or type 1 diabetes. J Clin Endocrinol Metab 2009; 94: 2464-2470.

41. Clausen TD, Mathiesen ER, Hansen T et al. High prevalence of type 2 diabetes and pre-diabetes in adult offspring of women with gestational diabetes mellitus or type 1 diabetes: the role of intrauterine hyperglycemia. Diabetes Care 2008; 31: $340-$ 346.

42. Murphy R, Ellard S \& Hattersley AT. Clinical implications of a molecular genetic classification of monogenic beta-cell diabetes. Nature Clin Pract 2008; 4: 200-213.

43. Lapolla A, Dalfra MG \& Fedele D. Diabetes related autoimmunity in gestational diabetes mellitus: is it important? Nutr Metab Cardiovasc Dis 2009; 19: 674-682.

44. Buchanan TA. Pancreatic B-cell defects in gestational diabetes: implications for the pathogenesis and prevention of type 2 diabetes. J Clin Endocrinol Metab 2001; 86: 989-993.

45. Catalano PM, Huston L, Amini SB et al. Longitudinal changes in glucose metabolism during pregnancy in obese women with normal glucose tolerance and gestational diabetes mellitus. Am J Obstet Gynecol 1999; 180: 903-916.

46. Catalano PM, Tyzbir ED, Roman NM et al. Longitudinal changes in insulin release and insulin resistance in nonobese pregnant women. Am J Obstet Gynecol 1991; 165: 1667-1672.

47. HAPO Study Cooperative Research Group. Hyperglycaemia and Adverse Pregnancy Outcome (HAPO) Study: associations with maternal body mass index. BJOG 2010; 117: 575-584.

48. Callaway LK, Prins JB, Chang AM et al. The prevalence and impact of overweight and obesity in an Australian obstetric population. Med J Aust 2006; 184: 56-59.

49. Moore LL, Singer MR, Bradlee ML et al. A prospective study of the risk of congenital defects associated with maternal obesity and diabetes mellitus. Epidemiology (Cambridge, Mass) 2000; 11: 689-694.

50. Bodnar LM, Siega-Riz AM, Simhan HN et al. Severe obesity, gestational weight gain, and adverse birth outcomes. Am J Clin Nutr 2010; 91: 1642-1648.

51. Flick AA, Brookfield KF, de la Torre L et al. Excessive weight gain among obese women and pregnancy outcomes. Am J Perinatol 2010; 27: 333-338.

52. Crane JM, White J, Murphy P et al. The effect of gestational weight gain by body mass index on maternal and neonatal outcomes. J Obstet Gynaecol Can 2009; 31: 28-35.

53. Hillier TA, Pedula KL, Vesco KK et al. Excess gestational weight gain: modifying fetal macrosomia risk associated with maternal glucose. Obstet Gynecol 2008; 112: 1007-1014.

54. Moran LJ, Misso ML, Wild RA et al. Impaired glucose tolerance, type 2 diabetes and metabolic syndrome in polycystic ovary syndrome: a systematic review and meta-analysis. Human Reproduction Update 2010; 16: 347-363.

55. Larter CZ, Chitturi S, Heydet D et al. A fresh look at NASH pathogenesis. Part 1: the metabolic movers. J Gastroenterol Hepatol 2010; 25: 672-690.

56. Alberti KG, Eckel RH, Grundy SM et al. Harmonizing the metabolic syndrome: a joint interim statement of the International Diabetes Federation Task Force on Epidemiology and Prevention; National Heart, Lung, and Blood Institute; American Heart Association; World Heart Federation; International Atherosclerosis Society; and International Association for the Study of Obesity. Circulation 2009; 120: 1640-1645.

57. Cornier MA, Dabelea D, Hernandez TL et al. The metabolic syndrome. Endocr Rev 2008; 29: 777-822.

58. Catalano PM. Obesity, insulin resistance and pregnancy outcome. Reproduction 2010; 140: 365-371.

59. Boden G. Obesity and free fatty acids. Endocrinol Metab Clin North Am 2008; 37: 635-646. viii-ix.

60. Kadowaki T, Yamauchi T, Kubota N et al. Adiponectin and adiponectin receptors in insulin resistance, diabetes, and the metabolic syndrome. J Clin Invest 2006; 116: 1784-1792.

61. Chatzi L, Plana E, Daraki V et al. Metabolic syndrome in early pregnancy and risk of preterm birth. Am J of Epidemiol 2009; 170: 829-836.

62. Mori T, Shinohara K, Wakatsuki A et al. Adipocytokines and endothelial function in preeclamptic women. Hypertens Res 2010; 33: 250-254.

63. Stekkinger E, Zandstra M. Peeters LL et al. Early-onset preeclampsia and the prevalence of postpartum metabolic syndrome. Obstet Gynecol 2009; 114: 1076-1084.

64. Retnakaran R, Qi Y, Connelly PW et al. Glucose intolerance in pregnancy and postpartum risk of metabolic syndrome in young women. J Clin Endocrinol Metab 2010; 95: 670-677.

65. Herrera E. Metabolic adaptations in pregnancy and their implications for the availability of substrates to the fetus. Eur J Clin Nutr 2000; 54(Suppl. 1): S47-S51.

66. Nolan CJ, Riley SF, Sheedy MT et al. Maternal serum triglyceride, glucose tolerance, and neonatal birth weight ratio in pregnancy. Diabetes Care 1995; 18: 1550-1556.

67. Koukkou E, Watts GF \& Lowy C. Serum lipid, lipoprotein and apolipoprotein changes in gestational diabetes mellitus: a cross-sectional and prospective study. J Clin Pathol 1996; 49: 634-637.

68. Hollingsworth DR \& Grundy SM. Pregnancy-associated hypertriglyceridemia in normal and diabetic women. Differences in insulin-dependent, non-insulin-dependent, and gestational diabetes. Diabetes 1982; 31: 1092-1097.

69. Ramsay JE, Ferrell WR, Crawford L et al. Maternal obesity is associated with dysregulation of metabolic, vascular, and inflammatory pathways. J Clin Endocrinol Metab 2002; 87: 4231-4237.

70. Chen X \& Scholl TO. Association of elevated free fatty acids during late pregnancy with preterm delivery. Obstet Gynecol 2008; 112: 297-303.

71. Schaefer-Graf UM, Graf K, Kulbacka I et al. Maternal lipids as strong determinants of fetal environment and growth in pregnancies with gestational diabetes mellitus. Diabetes Care 2008; 31: 1858-1863. 
72. Alvino G, Cozzi V, Radaelli $\mathrm{T}$ et al. Maternal and fetal fatty acid profile in normal and intrauterine growth restriction pregnancies with and without preeclampsia. Pediatr Res 2008; 64: 615-620.

73. O'Sullivan JB. Diabetes mellitus after GDM. Diabetes 1991; 40(Suppl. 2): 131-135.

74. Freinkel N. Banting Lecture 1980. Of pregnancy and progeny. Diabetes 1980; 29: 1023-1035.

75. Metzger BE, Buchanan TA, Coustan DR et al. Summary and recommendations of the Fifth International WorkshopConference on Gestational Diabetes Mellitus. Diabetes Care 2007; 30(Suppl. 2): S251-S260.

76. Jensen DM, Korsholm L, Ovesen P et al. Adverse pregnancy outcome in women with mild glucose intolerance: is there a clinically meaningful threshold value for glucose? Acta Obstet Gynecol Scand 2008; 87: 59-62.

77. Sermer M, Naylor CD, Gare DJ et al. Impact of increasing carbohydrate intolerance on maternal-fetal outcomes in 3637 women without gestational diabetes. The Toronto Tri-Hospital Gestational Diabetes Project. Am J Obstet Gynecol 1995; 173: 146-156.

78. Sacks DA, Greenspoon JS, Abu-Fadil S et al. Toward universal criteria for gestational diabetes: the 75-gram glucose tolerance test in pregnancy. Am J Obstet Gynecol 1995; 172: 607-614.

79. Rowan JA, Gao W, Hague WM et al. Glycemia and its relationship to outcomes in the metformin in gestational diabetes trial. Diabetes Care 2010; 33: 9-16.

80. American Diabetes Association. Standards of medical care in diabetes-2010. Diabetes Care 2010; 33(Suppl. 1): S11-S61.

81. Rowan JA, Hague WM, Gao W et al. Metformin versus insulin for the treatment of gestational diabetes. $N$ Engl J Med 2008; 358: 2003-2015.

82. Langer O, Conway DL, Berkus MD et al. A comparison of glyburide and insulin in women with gestational diabetes mellitus. N Engl J Med 2000; 343: 1134-1138.

83. Elliott BD, Langer O, Schenker S et al. Insignificant transfer of glyburide occurs across the human placenta. Am J Obstet Gynecol 1991; 165: 807-812.

84. Hebert MF, Ma X, Naraharisetti SB et al. Are we optimizing gestational diabetes treatment with glyburide? The pharmacologic basis for better clinical practice. Clin Pharmacol Therapeut 2009; 85: 607-614.

85. Kahn SE, Haffner SM, Heise MA et al. Glycemic durability of rosiglitazone, metformin, or glyburide monotherapy. $N$ Engl J Med 2006; 355: 2427-2443.

86. Schimmack G, Defronzo RA \& Musi N. AMP-activated protein kinase: role in metabolism and therapeutic implications. Diabetes Obes Metab 2006; 8: 591-602.

87. Zhou G, Myers R, Li Y et al. Role of AMP-activated protein kinase in mechanism of metformin action. J Clin Invest 2001; 108: 1167-1174.

88. Moses RG, Barker M, Winter M et al. Can a low-glycemic index diet reduce the need for insulin in gestational diabetes mellitus? a randomized trial. Diabetes Care 2009; 32: 996-1000.

89. Dornhorst A \& Frost G. The principles of dietary management of gestational diabetes: reflection on current evidence. J Hum Nutr Diet 2002; 15: 145-156. quiz 157-149.

90. Knowler WC, Barrett-Connor E, Fowler SE et al. Reduction in the incidence of type 2 diabetes with lifestyle intervention or metformin. N Engl J Med 2002; 346: 393-403.

91. Tuomilehto J, Lindstrom J, Eriksson JG et al. Prevention of type 2 diabetes mellitus by changes in lifestyle among subjects with impaired glucose tolerance. N Engl J Med 2001; 344: 1343-1350.

92. Buchanan TA, Xiang AH, Peters RK et al. Preservation of pancreatic beta-cell function and prevention of type 2 diabetes by pharmacological treatment of insulin resistance in high-risk Hispanic women. Diabetes 2002; 51: $2796-2803$.

93. Pan XR, Li GW, Hu YH et al. Effects of diet and exercise in preventing NIDDM in people with impaired glucose tolerance. The Da Qing IGT and Diabetes Study. Diabetes Care 1997; 20: 537-544.

94. Chiasson JL, Josse RG, Gomis R et al. Acarbose for prevention of type 2 diabetes mellitus: the STOP-NIDDM randomised trial. Lancet 2002; 359: 2072-2077. 